\title{
Reliability and validity of the Amharic version of European Organization for Research and Treatment of cervical Cancer module for the assessment of health related quality of life in women with cervical cancer in Addis Ababa, Ethiopia
}

Liya Teklu Araya', Gebremedhin Beedemariam Gebretekle ${ }^{1}$, Girma Tekle Gebremariam² and Teferi Gedif Fenta ${ }^{{ }^{*}}$

\begin{abstract}
Background: Cervical cancer is among the leading gynecological cancers affecting women worldwide. Maintenance and improvement of cervical cancer patients'health related quality of life (HRQoL) is an important issue. The cervical cancer specific quality of life module of the European Organization for Research and Treatment of Cancer (EORTC QLQCX24) is the most commonly used tool, however, it is not validated in Ethiopia. Hence, the present study aimed to assess the psychometric properties of the tool among Ethiopian cervical cancer patients.

Methods: Hospital based cross-sectional study was done in Tikur Anbessa Specialized Hospital (TASH), Addis Ababa, Ethiopia from January to February, 2018. The module was translated through forward-backward translation approach and pilot tested according to the EORTC Guidelines. One hundered and seventy one patients with confirmed cervical cancer were enrolled for the study. Amharic versions of EORTC QLQ-C30 and EORTC QLQ-CX24 were used to collect data along with socio-demographic and clinical characteristics. Descriptive statistics were used to assess sociodemographic and clinical characteristics of patients. The Psychometric properties of the EORTC QLQ-CX24 were evaluated in terms of acceptability, internal consistency, construct, concurrent and known group validity using SPSS version 22.

Results: One hundred seventy one cervical cancer patients were enrolled in the study, with a mean age of $52.15 \pm 10.4$ years. The EORTC QLQ-CX24 was found to be acceptable with high compliance and low missing responses. The Cronbach's alpha ranged from $0.70-0.84$, indicating the reliability of the scales. Convergent and discriminant validity in multitrait scaling analysis was adequate. The EORTC QLQ-C30 subscales and EORTC QLQ-CX24 subscales had a weak to strong correlation, indicating concurrent validity. The scales and single-item measures were able to discriminate between subgroups of patients differing with regard to performance status, cancer stage and treatment status, indicating clinical validity.
\end{abstract}

Conclusion: Amharic version of the EORTC QLQ-CX24 questionnaire is a valid and reliable tool and could be used for clinical and epidemiological cancer researches to study the HRQOL of patients with cervical cancer in Ethiopia.

Keywords: Cervical Cancer, HRQoL, Validation, Amharic version, EORTC QLQ-CX24

\footnotetext{
* Correspondence: tgedif@gmail.com; teferi.gedif@aau.edu.et

${ }^{1}$ Social and Administrative Pharmacy Unit, Department of Pharmaceutics and

Social Pharmacy,School of Pharmacy, College of Health Sciences, Addis

Ababa University, P.O. Box: 1176, Addis Ababa, Ethiopia

Full list of author information is available at the end of the article
}

(c) The Author(s). 2019 Open Access This article is distributed under the terms of the Creative Commons Attribution 4.0 International License (http://creativecommons.org/licenses/by/4.0/), which permits unrestricted use, distribution, and reproduction in any medium, provided you give appropriate credit to the original author(s) and the source, provide a link to the Creative Commons license, and indicate if changes were made. The Creative Commons Public Domain Dedication waiver (http://creativecommons.org/publicdomain/zero/1.0/) applies to the data made available in this article, unless otherwise stated. 


\section{Background}

Cervical cancer is the fourth most common cancer in women, and the seventh overall, with an estimated 528,000 new cases diagnosed every year. There were 266,000 deaths from cervical cancer worldwide, accounting for $7.5 \%$ of all female cancer deaths. An estimated $85 \%$ of the globally diagnosed cases and $88 \%$ of the deaths due to cervical cancer occur in developing nations [1]. Women in developing nations are at a 35\% greater lifetime risk of developing cervical cancer than women in high-income countries [2]; this is due to lack of early detection, screening and diagnosis of the disease at an advanced stage. Cervical cancer is most common in women older than 50 years. However, in developing nations, it is becoming increasingly prevalent among women during their reproductive age of 15-49 years [3]. In Ethiopia, cervical cancer is the second most prevalent cancer among women between 15 and 44 years of age. Every year, about 4648 new cases and 3235 deaths are reported [5]. Thus, maintenance and improvement of cervical cancer patients' health related quality of life (HRQoL) is becoming an important issue for health care providers in addition to tumor control and increasing survival rate of the patients [5].

HRQoL is a patient-reported outcome that is usually measured using carefully designed and validated instruments [5]. Although HRQoL of cancer patients has been well researched in developed countries, little is known about cancer patients in African countries, facing unique challenges such as poverty, access to health care and under resourced healthcare systems [6]. The European Organization for Research and Treatment of Cancer Quality of Life questionnaire (EORTC QLQ-C30) is a widely used instrument for assessing HRQoL of cancer patients [7]. Amharic is the official language of Ethiopia, spoken by most of the community and the Amharic version of the EORTC QLQ-C30 core questionnaire has been validated to assess $\mathrm{HRQoL}$ in Ethiopian cancer patients [8]. The English version of the EORTC QLQ-CX24 cervical cancer module is also available to measure quality of life in cervical cancer patients [9]. The psychometric properties of the EORTC QLQ-CX24 disease specific questionnaire was tested and established in many countries [9-11]. However, there is no cervical cancer specific assessment tool to measure quality of life with cervical cancer patients in Ethiopia. The diversity of culture between the western countries and Ethiopia could result in different interpretations of quality of life, so it is important to assess the acceptability, reliability and validity of the EORTC QLQ-CX24 as well as suitability for Ethiopian cervical cancer patients. Thus, the aim of the study was to assess the acceptability, reliability and validity of the cervical cancer disease specific EORTC QLQ-CX24 module.

\section{Methods}

\section{Design and participants}

A hospital based cross-sectional study was conducted among cervical cancer patients in Tikur Anebessa Specialized Hospital (TASH), Addis Ababa, Ethiopia from January to February, 2018. TASH is the sole oncology referral and radiotherapy center in the entire country. A total of 200 cervical cancer patients were requested for their participations. Participants were considered eligible if they had a pathologically diagnosed cervical cancer, were 18 years and older with the ability to understand and answer survey questions, and were able to speak and communicate well in the local (Amharic) language. Patients were excluded if they were unwilling to participate, unable to understand or complete the interview questionnaires or had a cognitive or mental problem.

\section{Instruments \\ EORTC QLQ-C30}

The 30-items EORTC QLQ-C30 is psychometrically robust, cross-culturally accepted and most frequently used tool to assess HRQoL in cancer patients. It is sub-grouped into 15 domains, including five functional subscales (physical functioning, role functioning, emotional functioning, cognitive functioning, and social functioning); three multi-item symptom subscales (fatigue, nausea/vomiting, and pain); global health status/QoL subscale; and six single items addressing various symptoms and perceived financial impact [7].

\section{EORTC QLQ-CX24}

The 24-items are classified into three multi-item scales, eleven items with symptom experience domain, three items with body image domain, and four items with sexual/ vaginal functioning domain. The other domains of the questionnaire are single-item scales, including lymphedema, peripheral neuropathy, menopausal symptom, sexual worry, sexual activity, and sexual enjoyment [9].

The standard scoring algorithm recommended by the EORTC was used to linearly transform all scales and item scores to a 0-100 scale; a high score for a functional scale represents a high/healthy level of functioning, whereas a high score for a symptom scale or item represents a high level of symptom or difficulties [12]. The EORTC QLQ-CX24 was translated by professional language speakers employed by the researchers into Amharic version through forward-backward translation approach according to the EORTC guideline [13]. The forward and backward translations were made by different professionals. Furthermore, it was pilot tested on 20 patients with cervical cancer in order to fix modifications to its wording. Thereafter, the authors assessed the EORTC QLQ-CX24 for its content validity as well its 
cultural adaptability and comprehensiveness in capturing all dimensions of HRQoL specific to cervical cancer.

\section{Data collection procedure}

Patients were enrolled during their visit at the oncology clinic of TASH and informed verbal consent was obtained from all patients. Data was collected by trained oncology nurses. Paper based Amharic versions of the EORTC QLQ-C30 and EORTC QLQ-CX24 module were read out loud for patients in a private room, as the majority of patients were unable to read and write. The data collectors completed the ECOG-PS and a questionnaire concerning sociodemographic and medical characteristics of the patients from the available medical records. Consent to use the English version of the EORTC QLQ-CX24 was obtained from the EORTC research group.

\section{Statistical analysis}

Descriptive statistics (frequency, percentage, mean with standard deviation) were used to report demographic and clinical characteristics. Psychometric properties of the EORTC QLQ-CX24 were assessed in terms of acceptability, internal consistency, construct validity, concurrent validity and known group validity.

The acceptability of the EORTC QLQ-CX24 was assessed in terms of response rate, percentage of missing data, time needed to complete the questionnaire and details of items considered upsetting, confusing or difficult in the questionnaire to respond. The internal consistency of each domains of the EORTC QLQ-CX24 was assessed using Cronbach's $\alpha$ coefficient, where $\alpha \geq$ 0.7 indicated adequate scale reliability of the tool [14].

The scale structure was evaluated by multi-trait scaling analysis $[10,15]$. This analysis was based on an evaluation of item-scale correlations for item-convergence and item-discriminance. Evidence of item-convergence was defined as a correlation of $(r \geq 0.40)$ between an item and its own scale. A lower correlation of an item with scales other than its own in comparison to the correlation with its own scale was evidence for item-discriminance. The convergent and discriminant validity for each scale was evaluated by assessing the Pearson's correlation coefficient. Scaling errors were defined as cases in which an item correlated "significantly less" with its own scale than with other scales. Known group validity was analyzed by independent t-test. This measured the extent to which the EORTC QLQ-CX24 scores were able to discriminate among the groups of different International Federation of Gynecology and Obstetrics (FIGO) stages [16], ECOG-PS [17], and patients being on treatment or not. Pearson correlation analysis was performed to explore the relationship between scales of the EORTC QLQ-C30 and the EORTC QLQ-CX24, this correlation aimed to assess if and to what extent clinical overlapping existed between the EORTC QLQ-C30 and EORTC QLQ-CX24 scales. All statistical procedures were performed using SPSS version 22 (SPSS, Inc. Chicago, USA). Significance level of $p<0.05$ was employed for the independent t- test and Pearson correlations.

\section{Results}

\section{Patient characteristics}

From the 200 cervical cancer patients, 171 were willing to participate and completed the questionnaires. All patients responded to both the EORTC QLQ-C30 and EORTC QLQ-CX24 without missing response. Majority $118(69 \%)$ of the patients had no formal education, 95 (56\%) of the patients were married and their mean age was $52.15 \pm 10.4$ years. Although the majority166 (97\%) of the patients were diagnosed with FIGO stage II-IV, 145 (85\%) of the respondents had a good performance status (ECOG-PS (0-1)). Among the patients, 158 (92.4\%) of them were admitted as an outpatient. More than half $(51 \%)$ of the patients were not receiving cancer treatment while 76 (44\%) of them were receiving radiotherapy. All patients had previously taken analgesics and had prescriptions to relieve symptoms while they waited their turn to receive cancer treatment (Table 1).

\section{Acceptability}

The EORTC QLQ-C30 and EORTCQLQ- CX24 were easily understood with full compliance. Of 200 patients, 171 volunteered to take part in the study and respond to the questionnaires without any missing response item. The total time for completion of the interview was approximately $10-15 \mathrm{~min}$, a maximum of $20 \mathrm{~min}$. Most patients $118(69.0 \%)$ reported that all questions are clear and easy to understand, even with uneducated individuals.

\section{Reliability and validity}

Reliability mainly indicates consistency of a measurement tool, ability to measure results in a consistent manner. Meanwhile validity refers to the extent to which a study actually captures or measures what it purports to examine. All of the scales had a Cronbach $\alpha \geq 0.70$. Correlation between the items within a scale indicated that all of the items of the EORTC QLQ-CX24 exhibited consistency. In terms of convergent validity, all items showed a good correlation with their own scales $(r \geq 0.4)$ and were highly correlated with their own scale than the other scales. There were no scaling errors found on the item-discriminance analysis, indicating the divergent validity (Table 2).

The correlation of an item and scales of the EORTC QLQ-CX24 with EORTC QLQ-C30 was used to assess 
Table 1 Socio-demographic and clinical characteristics of cervical cancer patients $(n=171)$

\begin{tabular}{|c|c|}
\hline Variables & Number of patients (\%) \\
\hline \multicolumn{2}{|l|}{ Religion } \\
\hline Orthodox & $112(65.5)$ \\
\hline Protestant & $35(20.5)$ \\
\hline Muslim & $24(14.0)$ \\
\hline \multicolumn{2}{|l|}{ Marital Status } \\
\hline Single & $2(1.2)$ \\
\hline Married & $96(56.1)$ \\
\hline Divorced & $30(17.5)$ \\
\hline Widowed & $43(25.1)$ \\
\hline \multicolumn{2}{|l|}{ Education } \\
\hline Unable to read and write & $118(69)$ \\
\hline Can read and write & $10(5.8)$ \\
\hline Primary education & $22(12.9)$ \\
\hline Secondary education & $14(8.2)$ \\
\hline \multicolumn{2}{|l|}{$\mathrm{FIGO}$} \\
\hline Stage 1 & $6(3.5)$ \\
\hline Stage 2 & $57(33.3)$ \\
\hline Stage 3 & $49(28.7)$ \\
\hline Stage 4 & $59(34.5)$ \\
\hline \multicolumn{2}{|l|}{ Patient status } \\
\hline New Admission & $46(26.9)$ \\
\hline Follow up & $125(73.1)$ \\
\hline \multicolumn{2}{|l|}{ Admission type } \\
\hline Inpatient & $13(7.6)$ \\
\hline Outpatient & $158(92.4)$ \\
\hline \multicolumn{2}{|l|}{ Current treatment } \\
\hline Surgery & $2(1.2)$ \\
\hline Chemotherapy & $5(2.9)$ \\
\hline Radiotherapy & $76(44.4)$ \\
\hline Not on treatments & $88(51.5)$ \\
\hline \multicolumn{2}{|l|}{ ECOG-PS } \\
\hline 0 & $1(0.6)$ \\
\hline 1 & $144(84.2)$ \\
\hline 2 & $19(11.1)$ \\
\hline 3 & $7(4.1)$ \\
\hline
\end{tabular}

SD Standard Deviation, FIGO International Federation Gynaecology and Obstetrics, ECOG-PS European Cooperative Oncology Group Performance Status; $\mathrm{n}=$ Sample size

the concurrent validity. Correlation between the EORTC QLQ-CX24 and the EORTC QLQ-C30 scales are presented in Table 3. Scales and items of the EORTC QLQ-CX24 were found to be correlated, to some extent, with the scales of the EORTC QLQ-C30. This indicated that the EORTC QLQ-CX24 partially assessed a unique set of HRQoL domains that are currently not covered by the core questionnaire. The symptom experience, body image, peripheral neuropathy and menopausal symptom scales correlated strongly with the EORTC QLQ-C30 functioning scales $(r \geq 0.40)$. The symptom scales of the EORTC QLQ-CX24 strongly correlated with all subscales of the EORTC QLQ-C30, except for diarrhea and financial impact. The menopausal symptom scale indicated a strong negative correlation with the physical functioning, emotional functioning and social functioning. On the other hand, scales that were related to sexuality (the sexual/vaginal functioning, sexual worry, and sexual enjoyment scales) were weakly correlated with the EORTC QLQ-C30 $(r \leq 0.4)$.

Known group validity was established by assessing the ability of the EORTC QLQ-CX24 to detect difference in level of HRQoL across patient's performance status, whether patients being on treatments or not, and FIGO stages. Results differed significantly among patients' performance status with lymphedema $(p<0.05)$ and the remaining functioning and symptom scales had no significant difference in the HRQoL among the different ECOG-PS. The sexual/vaginal functioning mean score showed a difference among treatment groups. Although not significant, differences in mean score were observed among the groups and scales \& items of the EORTC QLQ-CX24.

The study showed that symptom experience, sexual/ vaginal functioning scales and sexual activity items were capable of discriminating among patients with different FIGO stages $(p<0.05)$. No significant difference of mean scores for patients with different FIGO stages was found in other functioning and symptom scales of the EORTC QLQ-CX24 (Table 4). For instance, mean scores of the symptom experience, peripheral neuropathy, menopausal symptom and sexual worry of patients with progressive cervical cancer (Stages II-IV) were higher than those with early cervical cancer (Stages I-II). On the other hand, the result for sexual enjoyment and sexual activity showed that as the stage progress, the mean scores become lower, indicating a lower quality of life as well.

\section{Discussion}

This is the first study conducted to establish if the Amharic version of EORTC QLQ-CX24 questionnaire is an acceptable, reliable and valid measure to assess HRQoL in Ethiopian cervical cancer patients. The finding of the study revealed that the Amharic version of EORTC QLQ-CX24 has good acceptability and is psychometrically robust tool to measure HRQoL for Ethiopian cervical cancer patients.

All presented Cronbach alpha values exceeded the 0.7 criterion. The internal consistency of the body image scale in this study was found to be consistent with 
Table 2 Multi-trait scaling analysis with Pearson correlation between scale items on the EORTC QLQ-CX24

\begin{tabular}{|c|c|c|c|c|c|}
\hline \multirow{2}{*}{$\frac{\text { EOTC QLQ-CX24 }}{\text { Symptom scales }}$} & \multicolumn{4}{|c|}{ Item/s number Mean \pm SD Cronbach alpha Convergent validity ${ }^{a}$ Discriminant validity ${ }^{b}$} & \\
\hline & & & & & \\
\hline Symptom experience & $31-37,39,41-43$ & $42.97(23.68)$ & 0.84 & $0.50-0.78$ & $0.01-0.63$ \\
\hline Body image & $45-47$ & $45.22(39.57)$ & 0.96 & $0.95-0.97$ & $0.16-0.65$ \\
\hline Sexual/Naginal function & $50-53$ & $64.47(28.84)$ & 0.70 & $0.62-0.88$ & $0.02-0.79$ \\
\hline \multicolumn{6}{|l|}{ Symptom items } \\
\hline Lymphedema & 38 & $12.86(27.83)$ & N/A & N/A & $0.06-0.33$ \\
\hline Peripheral neuropathy & 40 & $40.54(39.02)$ & N/A & N/A & $0.05-0.49$ \\
\hline Menopausal symptoms & 44 & $54.77(37.50)$ & N/A & N/A & $0.01-0.49$ \\
\hline Sexual worry & 48 & $33.52(46.23)$ & N/A & N/A & $0.10-0.70$ \\
\hline \multicolumn{6}{|l|}{ Functional Items } \\
\hline Sexual activity & 49 & $7.49(22.33)$ & N/A & N/A & $0.20-0.39$ \\
\hline Sexual enjoyment & 54 & $38.59(31.93)$ & N/A & N/A & $0.06-0.39$ \\
\hline
\end{tabular}

$S D$ standard deviation, N/A not applicable; ${ }^{a}$ Item-own scale correlation using Pearson correlation coefficient; ${ }^{b}$ Item-other scale correlation, Pearson correlation coefficient

previous studies $[9,18]$. Pearson correlation analysis confirmed the satisfactory convergent and discriminant validity of the EORTC QLQ-CX24, as correlations had $r \geq 0.40$. Similar to the English version, symptom scale, body image and vaginal functioning have good item convergence [9], which showed that each item was highly correlated with one another as compared to items of another scale. All the items have good discriminant validity except for item 53 of the vaginal functioning scale, which ask about pain during sexual intercourse, having exhibited a higher positive correlation with sexual worry.

Sexual/vaginal functioning and sexual enjoyment were conditional; a patient could skip answering the question if they have not had sexual activity in the past 4 weeks. As a result, $152(89 \%)$ patients skipped the sexual/vaginal functioning scale and sexual enjoyment item. This indicated that the status of the patients highly influenced their sexual activity and functioning. Another studies

Table 3 Pearson correlation between EORTC QLQ-CX24 and EORTC QLQ-C30

\begin{tabular}{|c|c|c|c|c|c|c|c|c|c|}
\hline \multirow[t]{2}{*}{ EORTC QLQ-C30 } & \multicolumn{9}{|c|}{ EORTC QLQ-CX24 } \\
\hline & $\begin{array}{l}\text { Symptom } \\
\text { experience }\end{array}$ & $\begin{array}{l}\text { Body } \\
\text { image }\end{array}$ & $\begin{array}{l}\text { Sexual /vaginal } \\
\text { functioning }\end{array}$ & Lymphedema & $\begin{array}{l}\text { Peripheral } \\
\text { neuropathy }\end{array}$ & $\begin{array}{l}\text { Menopausal } \\
\text { symptoms }\end{array}$ & $\begin{array}{l}\text { Sexual } \\
\text { worry }\end{array}$ & $\begin{array}{l}\text { Sexual } \\
\text { activity }\end{array}$ & $\begin{array}{l}\text { Sexual } \\
\text { enjoyment }\end{array}$ \\
\hline \multicolumn{10}{|l|}{ Functioning scales } \\
\hline Physical Functioning & $-0.56^{\mathrm{b}}$ & $0.42^{b}$ & 0.40 & $-0.20^{b}$ & $-0.42^{b}$ & $-0.43^{b}$ & -0.14 & -0.12 & -0.10 \\
\hline Role Functioning & $-0.49^{b}$ & $0.42^{\mathrm{b}}$ & 0.07 & -0.12 & $-0.40^{\mathrm{b}}$ & $-0.35^{b}$ & -0.15 & -0.11 & -0.08 \\
\hline Emotional Functioning & $-0.45^{\mathrm{b}}$ & $0.49^{b}$ & 0.31 & -0.14 & $-0.31^{b}$ & $-0.40^{b}$ & -0.14 & $-0.23^{b}$ & 0.03 \\
\hline Cognitive Functioning & $-0.33^{b}$ & $0.35^{b}$ & 0.17 & $-0.29^{b}$ & $-0.25^{b}$ & $-0.20^{b}$ & -0.15 & -0.11 & -0.08 \\
\hline Social Functioning & $-0.48^{b}$ & $0.46^{\mathrm{b}}$ & 0.43 & -0.11 & $-0.39^{b}$ & $-0.43^{b}$ & -0.08 & -0.01 & -0.19 \\
\hline \multicolumn{10}{|l|}{ Symptom scales } \\
\hline Fatigue & $0.58^{\mathrm{b}}$ & $0.36^{b}$ & -0.02 & 0.14 & $0.31^{b}$ & $0.34^{\mathrm{b}}$ & 0.09 & 0.05 & 0.14 \\
\hline Nausea and Vomiting & $0.31^{b}$ & -0.15 & -0.28 & 0.02 & $0.18^{\mathrm{a}}$ & $0.18^{\mathrm{a}}$ & 0.11 & 0.70 & -0.11 \\
\hline Pain & $0.64^{b}$ & $0.32^{b}$ & -0.20 & 0.13 & $0.38^{b}$ & $0.42^{\mathrm{b}}$ & 0.01 & -0.01 & -0.15 \\
\hline Dyspnea & $0.50^{\mathrm{b}}$ & $0.23^{b}$ & -0.28 & $0.20^{\mathrm{a}}$ & $0.17^{\mathrm{a}}$ & $0.28^{b}$ & 0.04 & 0.03 & 0.10 \\
\hline Insomnia & $0.40^{\mathrm{b}}$ & $0.26^{b}$ & 0.18 & 0.07 & $0.22^{b}$ & $0.23^{b}$ & 0.05 & -0.03 & 0.15 \\
\hline Loss of appetite & $0.45^{\mathrm{b}}$ & $0.21^{b}$ & 0.01 & 0.06 & $0.34^{b}$ & $0.28^{\mathrm{b}}$ & -0.01 & -0.04 & $-0.49^{a}$ \\
\hline Constipation & $0.65^{\mathrm{b}}$ & $0.33^{b}$ & -0.26 & 0.13 & $0.23^{b}$ & $0.30^{\mathrm{b}}$ & 0.09 & -0.04 & 0.17 \\
\hline Diarrhea & 0.07 & -0.04 & $-0.56^{\mathrm{a}}$ & 0.11 & $0.18^{\mathrm{a}}$ & -0.01 & -0.04 & -0.11 & 0.39 \\
\hline Financial difficulties & $0.19^{\mathrm{a}}$ & $0.29^{b}$ & 0.03 & $0.15^{\mathrm{a}}$ & $0.20^{\mathrm{b}}$ & $0.21^{b}$ & 0.10 & -0.08 & -0.12 \\
\hline Global health status/ QoL & $-0.64^{b}$ & $0.40^{b}$ & $0.53^{\mathrm{a}}$ & -0.14 & $-0.33^{b}$ & $-0.35^{b}$ & -0.06 & -0.01 & -0.27 \\
\hline
\end{tabular}

${ }^{a}$ Correlation is significant at the $\mathrm{p} 0.05$ level; ${ }^{\mathrm{b}}$ Correlation is significant at the 0.01 level 
Table 4 Comparisons between EORTC QLQ-CX24 scores in different FIGO stages, performance status and treatment groups

\begin{tabular}{|c|c|c|c|c|c|c|}
\hline \multirow[t]{2}{*}{ EORTC QLQ-CX24 } & \multicolumn{2}{|l|}{ ECOG-PS } & \multicolumn{2}{|l|}{ FIGO stages } & \multicolumn{2}{|c|}{ Treatment groups } \\
\hline & $\begin{array}{l}\text { Good PS (0-1) } \\
(n=145)\end{array}$ & $\begin{array}{l}\text { Poor PS [2-4] } \\
(n=26)\end{array}$ & $\begin{array}{l}\text { Stage I- } \| \\
(n=63)\end{array}$ & $\begin{array}{l}\text { Stage III-IV } \\
(n=108)\end{array}$ & $\begin{array}{l}\text { On treatment } \\
(n=83)\end{array}$ & $\begin{array}{l}\text { Off treatment } \\
(n=88)\end{array}$ \\
\hline Symptom experience & $42.40(24.40)$ & $46.15(19.22)$ & $38.72(24.20)^{a}$ & $45.45(23.12)^{a}$ & $40.67(25.46)$ & $45.14(21.78)$ \\
\hline Body image & $46.89(39.35)$ & $35.89(40.28)$ & $45.50(41.65)$ & $45.06(38.51)$ & $47.25(39.02)$ & $43.30(40.22)$ \\
\hline Sexual /vaginal functioning & $63.88(29.56)$ & $75.00(0.00)$ & $65.27(29.26)^{a}$ & $63.09(30.37)^{\mathrm{a}}$ & $54.62(33.87)$ & $73.33(21.44)$ \\
\hline Lymphedema & $10.57(24.75)^{a}$ & $25.64(39.22)^{a}$ & $17.98(31.00)$ & $9.87(25.47)$ & $16.86(32.24)^{a}$ & $9.09(22.4)^{a}$ \\
\hline Peripheral neuropathy & $40.22(39.06)$ & $42.30(39.50)$ & $42.32(38.43)$ & $39.50(39.50)$ & $44.97(39.79)$ & $36.36(38.0)$ \\
\hline Menopausal symptoms & $53.79(37.92)$ & $60.25(35.30)$ & $53.43(39.04)$ & 55.55 (36.74) & $53.81(37.83)$ & $55.68(37.38)$ \\
\hline Sexual worry & $32.41(46.13)$ & $39.74(47.15)$ & $39.15(47.34)$ & $30.24(45.46)$ & $32.53(44.77)$ & $34.46(47.79)$ \\
\hline Sexual activity & $8.39(23.55)$ & $2.56(13.07)^{\mathrm{a}}$ & $11.82(26.37)$ & $4.98(19.31)$ & $8.13(24.59)$ & $6.89(20.75)$ \\
\hline Sexual enjoyment & 37. 03 (32.11) & $66.66(0.00)$ & $33.33(24.61)$ & $47.61(42.41)$ & $40.74(32.39)$ & $36.66(33.14)$ \\
\hline
\end{tabular}

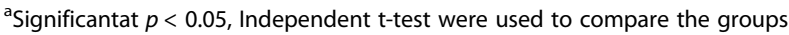

also indicated that cultural boundaries and privacy of the sexuality could have an influence in HRQoL $[11,19]$.

The Pearson correlations between the EORTC QLQ-C30 and the EORTC QLQ-CX24 were strong in the majority of the scales. In the Chinese and Polish validation studies [10, 19], the EORTC QLQ-CX24 scores strongly correlated with the EORTC QLQ-C30 core questionnaires, indicating that those scales that assess similar aspects were strongly correlated. Weak or no correlation indicated that the EORTC QLQ-CX24 has unique domains of HRQoL, which are not addressed by the EORTC QLQ-C30. These results are another confirmation on the need to conjugate the cervical cancer specific questionnaire with the general cancer questionnaire in order to assess the HRQoL of a patient with cervical cancer.

The result of the known group validity showed that the EORTC QLQ-CX24 cervical cancer specific module was able to discriminate between patients with different FIGO disease stages and ECOG-PS as well as patients being on treatment or not. The symptom scale differentiates between the early and advanced FIGO stages of cervical cancer.

The lymphedema single item differentiated among the good and poor ECOG-PS of the patients. This is quite different result compared to the English version, which found that symptom experience differentiated among all FIGO stages and treatment options. Other studies shared a similar result with only one sub-scale differentiating between the FIGO stages [10, 19, 20]. The current finding differed from others and such difference might be attributed to differences in the socio-demographics, comorbidities, perceptions about the disease, culture and healthcare service of the hospitals [9]. Only 10\% of the patients responded to question about sexual functioning and enjoyment. Since the number of sexually active patients was very small, the significance of the correlation of the scale and items could not be established as well as generalized to the sample size. Furthermore, the response to sexual activity might be attributed to the cultural hindrance regarding sexuality of a woman $[9,20]$. In addition, it has been documented that any disease condition of the "sexual area" would influence the patient's body image and sexual worry regardless of the stages $[10,11]$.

Finally, this validation study had certain limitations. The study did not measure test-retest reliability and the responsiveness of the EORTC QLQ-CX24. In addition, because of limited number of sexually active patients, the actual correlation of the scales could not be indicated and further research with larger sample size is recommended.

\section{Conclusions}

Amharic version of the EORTC QLQ-CX24 questionnaire is a valid and reliable tool and could be used in clinical and epidemiological cancer researches to study the HRQoL of patients with cervical cancer in Ethiopia.

\section{Abbreviations}

ECOG-PS: European Cooperative Oncology Group Performance status; EORTC QLQ-C30: European Organization for Research and Treatment of Cancer

Quality of life Questionnaire version 3; EORTC QLQ-CX24: European Organization for Research and Treatment of Cancer Quality of Life Questionnaire cervical cancer specific module; FIGO: International Federation of Gynaecology and Obstetrics; HRQoL: Health Related Qaulity of life.; TASH: Tikur Anebessa Specialized Hospital.

\section{Acknowledgments}

The authors would like to thank Addis Ababa University for providing financial support for carrying out this study. We would like to extend our sincere thanks to all research participants as well as other institutions that supported the study.

\section{Funding}

Addis Ababa University funded this study for partial fulfillment of Master's degree in Pharmacoepidemiology and Social pharmacy to the first Author. 


\section{Availability of data and materials}

The dataset is not publicly available due to required data protection, but available upon reasonable request with a signature of data privacy form. To request the data, the reader may contact the authors through email. The dataset is available from the first author on reasonable request.

\section{Authors' contributions}

LTA and GBG conceived the research idea, did translation and transcultural adaptation of the EORTC-QLQ-CX24; LTA, GBG, GTG and TGF performed data analysis and interpretation; LTA write original draft of the manuscript; LTA, GTG, GBG and TGF reviewed and edited the manuscript. All authors critically evaluated the final manuscript for important intellectual content and approved the final version of the manuscript.

\section{Ethics approval and consent to participate}

The study obtained approval from the Ethics Review Committee of the School of Pharmacy, Collage of Health Sciences, Addis Ababa University and Institutional Review Board of TASH. In addition, written informed consent was obtained from patients.

\section{Consent for publication}

Not applicable.

\section{Competing interests}

The authors declare that they have no competing interests.

\section{Publisher's Note}

Springer Nature remains neutral with regard to jurisdictional claims in published maps and institutional affiliations.

\section{Author details}

${ }^{1}$ Social and Administrative Pharmacy Unit, Department of Pharmaceutics and Social Pharmacy,School of Pharmacy, College of Health Sciences, Addis Ababa University, P.O. Box: 1176, Addis Ababa, Ethiopia. ${ }^{2}$ Department of Pharmacology and Clinical Pharmacy, School of Pharmacy, College of Health Sciences, Addis Ababa University, Addis Ababa, Ethiopia.

Received: 5 July 2018 Accepted: 10 January 2019

Published online: 14 January 2019

\section{References}

1. International Agency for Research on Cancer. All Cancers Estimated Incidence, Mortality and Prevalence of All Cancers (excluding nonmelanoma skin cancer) in 2012. J Natl Cancer Inst. 2017;(109):9. https://doi. org/10.1093/jnci/djx205.

2. Echelman D, Feldman S. Management of cervical precancers: a global perspective. Hematol Oncol Clin North Am. 2012;26(1):31-44.

3. Catarino R, Petignat P, Dongui G, Vassilakos P. Cervical cancer screening in developing countries at a crossroad: emerging technologies and policy choices Catarino $R$ et al Screening for cervical cancer screening in developing countries. World J Clin Oncol. 2015;6(6):281-90.

4. Hailemariam T, Yohannes B, Aschenaki H, Mamaye E, Orkaido G, Seta M. Prevalence of cervical Cancer and associated risk factors among women attending cervical Cancer screening and diagnosis Center at Yirgalem General Hospital, southern Ethiopia. J Cancer Sci Ther. 2017;9(11):730-5.

5. Velikova G, Stark D, Selby P. Quality of life instruments in oncology. Eur J Cancer. 1999;35(11):1571-80.

6. Van Rensburg JJ, Maree JE, Casteleijn D. An investigation into the quality of life of cancer patients in South Africa. Asia-Pacific J Oncol Nurs. 2017; 4(4):336-41.

7. Aaronson NK, Ahmedzai S, Bergman B, Bullinger M, Cull A, Duez NJ, et al. The European Organization for Research and Treatment of Cancer QLQ-C30: a quality-of-life instrument for use in international clinical trials in oncology. J Natl Cancer Inst. 1993;85(5):365-76.

8. Ayana BA, Negash S, Yusuf L, Tigeneh W, Haile D. Reliability and validity of amharic version of EORTC QLQ-C30 questionnaire among gynecological cancer patients in Ethiopia. PLoS One. 2016;11(6):1-10.

9. Greimel ER, Vlasic KK, Waldenstrom AC, Duric VM, Jensen PT, Singer S, et al. The European Organization for Research and Treatment of Cancer (EORTC) quality-of-life questionnaire cervical cancer module: EORTC QLQ-CX24. Cancer. 2006;107(8):1812-22.
10. Hua C, Guo H-M, Guan X, Kong F-J, Hou R-J, Zhang X-Y, et al. Validation of the european organization for research and treatment of cancer cervical cancer module for chinese patients with cervical cancer. Dovepress. 2013;7: 1061-6.

11. Jayasekara H, Rajapaksa LC, Greimel ER. The EORTC QLQ-CX24 cervical cancer-specific quality of life questionnaire: psychometric properties in a south Asian sample of cervical cancer patients. Psychooncology. 2008; 17(10):1053-7.

12. Fayers PM, Aaronson NK, Bjordal K, Groenvold M, Curran D, Bottomley A, on behalf of the European Organisation for Research and Treatment of Cancer Quality of Life Study Group. The EORTC QLQ-C30 Scoring Manual (3rd edn). Brussels: EORTC; 2001.

13. Beaton DE, Bombardier C, Guillemin F, Ferraz MB. Guidelines for the process of cross-cultural adaptation of self-report measures. Spine. 2000;25(24):3186-91.

14. Cronbach LJ. Coefficient alpha and the internal structure of tests. Psychometrika. 1951;16(3):297-334.

15. Shin DW, Ahn E, Kim YM, et al. Cross-cultural application of the Korean version of the European Organization for Research and Treatment of Cancer quality of life questionnaire cervical cancer module. Oncology. 2009;76(3): 190-8.

16. Pecorelli S. Revised FIGO staging of carcinoma of the vulva, cervix, and endometrium. Int J Gynecol Obstet. 2009:105:103-4.

17. Conill C, Verger E, Salamero M. Performance status assessment in cancer patients. Cancer. 1990;65(8):1864-6.

18. Ayasekara H, Rajapaksa LC, Greimel ER. The EORTC QLQ-CX24 cervical cancer-specifc quality of life questionnaire: psychometric properties in a south Asian sample of cervical cancer patients. Psychooncology. 2008; 17(10):1053-7.

19. Paradowska D, Tomaszewski KA, Bałajewicz-Nowak M, Bereza K, Tomaszewska IM, Paradowski J, et al. Validation of the polish version of the EORTC QLQ-CX24 module for the assessment of health-related quality of life in women with cervical cancer. Eur J Cancer Care. 2014;23(2):214-20.

20. Du Toit GC, Nel DG. Translation and validation of the EORTC QLO-CX24 questionnaire into the indigenous African languages of isiXhosa and Afrikaans. South Afr J Gynaecol Oncol. 2012;4(2):59-62.
Ready to submit your research? Choose BMC and benefit from:

- fast, convenient online submission

- thorough peer review by experienced researchers in your field

- rapid publication on acceptance

- support for research data, including large and complex data types

- gold Open Access which fosters wider collaboration and increased citations

- maximum visibility for your research: over $100 \mathrm{M}$ website views per year

At $\mathrm{BMC}$, research is always in progress.

Learn more biomedcentral.com/submissions 